\title{
Cell detachment activates the Hippo pathway via cytoskeleton reorganization to induce anoikis
}

\author{
Bin Zhao, ${ }^{1,2} \mathrm{Li} \mathrm{Li}^{2}{ }^{2}$ Lloyd Wang, ${ }^{2}$ Cun-Yu Wang, ${ }^{3}$ Jindan $\mathrm{Yu}^{,}{ }^{4}$ and Kun-Liang Guan ${ }^{2,5}$ \\ ${ }^{1}$ Life Sciences Institute, Zhejiang University, Hangzhou, Zhejiang 310058, China; ${ }^{2}$ Department of Pharmacology, Moores Cancer \\ Center, University of California at San Diego, La Jolla, California 92093, USA; ${ }^{3}$ Laboratory of Molecular Signaling, Division of Oral \\ Biology and Medicine, University of California at Los Angeles School of Dentistry, Los Angeles, California 90095, USA; \\ ${ }^{4}$ Department of Medicine, Robert H. Lurie Comprehensive Cancer Center, Northwestern University, Chicago, Illinois 60611, USA
}

Cell attachment to the extracellular matrix (ECM) is crucial to cell physiology such as polarity, motility, and proliferation. In normal cells, loss of attachment to the ECM induces a specific type of apoptosis, termed anoikis. Resistance to anoikis in cancer cells promotes their survival in circulation and dispersion to distant anatomic sites, leading to tumor metastasis. The Yes-associated protein (YAP) transcription coactivator is a human oncogene and a key regulator of organ size. The Hippo tumor suppressor pathway phosphorylates and inhibits YAP. However, little is known about the signals that regulate the Hippo pathway. Here we report that through cytoskeleton reorganization, cell detachment activates the Hippo pathway kinases Lats1/2 and leads to YAP phosphorylation and inhibition. The detachment-induced YAP inactivation is required for anoikis in nontransformed cells, whereas in cancer cells with deregulation of the Hippo pathway, knockdown of YAP and TAZ restores anoikis. Furthermore, we provided evidence that Lats $1 / 2$ expression level is indeed significantly downregulated in metastatic prostate cancer. Our findings provide a novel connection between cell attachment and anoikis through the Hippo pathway and have important implications in cancer therapeutics.

[Keywords: YAP; Lats; phosphorylation; anoikis; cancer metastasis]

Supplemental material is available for this article.

Received June 30, 2011; revised version accepted November 23, 2011.

In multicellular organisms, the size of each organ is strictly regulated. This process involves many signaling pathways, and their dysregulation can lead to uncontrolled growth and cancer (Hanahan and Weinberg 2000). The Hippo pathway is a newly established tumor suppressor pathway that limits organ size under physiological conditions (Justice et al. 1995; Xu et al. 1995; Kango-Singh et al. 2002; Tapon et al. 2002; Harvey et al. 2003; Jia et al. 2003; Pantalacci et al. 2003; Udan et al. 2003; Wu et al. 2003; Huang et al. 2005; Lai et al. 2005; Dong et al. 2007). At the core of the Hippo pathway is a kinase cascade consisting of Mst1/2 and Lats1/2. Mst1/2 kinases (Drosophila Hippo homologs) complex with the scaffold protein Sav1 to phosphorylate and activate the Lats1/2 kinases, which complex with another scaffold protein, Mob1 (Halder and Johnson 2011). Lats1/2 directly phosphorylate Yes-associated protein (YAP) on serine residues in five consensus HXRXXS motifs (Zhao et al. 2010). Phosphorylation of YAP S127 generates a 14-3-3-binding motif responsible for YAP cytoplasmic retention (Zhao et al. 2007; Hao et al. 2008).

${ }^{5}$ Corresponding author.

E-mail kuguan@ucsd.edu.

Article is online at http://www.genesdev.org/cgi/doi/10.1101/gad.173435.111.
Thereby, YAP is inhibited by a phosphorylation-induced physical separation from nuclear-localized target transcription factors and target gene promoters. Furthermore, phosphorylation of YAP S381 by Lats1/2 promotes YAP ubiquitination and degradation (Zhao et al. 2010). TAZ, the YAP paralog, is inhibited by the Hippo pathway through similar mechanisms (Lei et al. 2008; Liu et al. 2010).

Upstream signals that regulate the Hippo pathway are largely unknown. We previously reported that cell-cell contact and high cell density activate the Hippo pathway to inhibit YAP (Zhao et al. 2007). Further studies demonstrated that cell-cell junctional proteins such as the angiomotin protein complex and $\alpha$-catenin inhibit YAP (Nishioka et al. 2009; Varelas et al. 2010; Chan et al. 2011; Kim et al. 2011; Schlegelmilch et al. 2011; Silvis et al. 2011; Wang et al. 2011; Zhao et al. 2011). In addition to cell-cell contact, cells also physically interact with the extracellular matrix (ECM) in vivo. For epithelial cells, the interaction of basal plasma membrane with the ECM leads to a drastic effect on cell shape, polarity, motility, survival, and proliferation (Frantz et al. 2010). In this study, we provide evidence that cell detachment activates the Hippo pathway kinases Lats1/2 to inhibit YAP. More im- 
portantly, this YAP inactivation is required for detachmentinduced anoikis. Consistent with these findings, Lats $1 / 2$ expression is repressed in metastatic prostate cancer. In addition, actin and microtubule organization mediates Lats $1 / 2$ activation in response to cell detachment. Thus, our findings provide new insights into the mechanism of anoikis through the Hippo pathway-mediated YAP inhibition evoked by cell detachment and a possible role of this regulation in cancer metastasis.

\section{Results}

YAP phosphorylation, localization, and activity are regulated by cell attachment to the ECM

In order to determine whether the Hippo pathway could be regulated by cell-ECM contact, we examined the effect of cell attachment on YAP phosphorylation. Interestingly, during cell attachment, YAP exhibited a dramatic dephosphorylation, as indicated by a phospho-specific antibody and an increased mobility on Phos-tag-containing SDS-PAGE gels (Fig. 1A), which is a useful tool for detecting protein phosphorylation via mobility shift. Consistently, when cells were detached by trypsinization (T), YAP became phosphorylated within $10 \mathrm{~min}$ (Fig. 1B). The phosphorylation nature of the YAP mobility shift was confirmed by $\lambda$ protein phosphatase treatment, which converted YAP to the faster-migrating form (Fig. 1B). In addition, cell detachment by an enzyme-free cell dissociation method also leads to YAP phosphorylation, excluding the possibility of YAP phosphorylation as a result of trypsin cleavage of cell surface molecules (Supplemental Fig. S1A). YAP phosphorylation by Lats $1 / 2$ kinases of the Hippo pathway is known to cause cytoplasmic translocation (Zhao et al. 2007). Consistently, when MCF10A cells were attached for 10 min and YAP phosphorylation remained high (Fig. 1A), we observed YAP to be mainly in the cytoplasm (Fig. 1C). However, after cells were attached for $80 \mathrm{~min}$, at which time YAP phosphorylation was low, we found YAP to be mainly localized in the nucleus. Therefore, our results suggest that cell attachment and detachment modulate both YAP phosphorylation and subcellular localization.

Consistent with a functional importance of nuclear YAP, cell attachment also regulates YAP target gene expression. When cells were attached for longer periods of time, direct YAP target genes such as CTGF (Zhao et al. 2008) were strongly induced (Fig. 1D,E). However, when cells were plated onto ultralow attachment plates, they remained in suspension and there was no induction of CTGF expression. Similarly, by examining mRNA levels, we also observed the induction of additional YAP target genes such as Cyr61 (Zhang et al. 2011), Inhba (Zhao et al. 2007), and Ankrd1 (Zhao et al. 2008) in attached but not suspended cells (Fig. 1E). Taken all together, these data indicate that cell-ECM attachment leads to YAP dephosphorylation, nuclear translocation, and activation. In contrast, cell detachment results in YAP phosphorylation, cytoplasmic translocation, and inhibition.
Focal adhesion (FA) is not required for cell attachment-induced activation of YAP

Cell-ECM interaction alters cell architecture and physiology in many ways, including FA formation and cytoskeleton reorganization (Frantz et al. 2010). FAs are complex intracellular linkages between integrins and the F-actin cytoskeleton, which function as a mechanical force transducer and a signaling module (Parsons et al. 2010). To address whether formation of FAs plays a role in the activation of YAP by cell attachment, we compared cells in attachment to fibronectin, a FA-inducing integrin ligand, or polylysine, which does not organize FA /Grashoff et al. 2010). Within the initial $10 \mathrm{~min}$ of cell adherence to fibronectin-coated coverglasses, FAs were not observed and YAP was mainly localized to the cytoplasm (Fig. 1F). At later time points, FAs gradually formed as indicated by clustering of the FA protein vinculin (Fig. 1F). Notably, YAP nuclear localization occurred faster than FA formation (Fig. 1F, 1-h and 2-h time points). More importantly, cells that were attached to polylysine-coated coverglasses also showed a dramatic YAP translocation to the nucleus, although those cells did not form FAs (Fig. 1F). Furthermore, cell attachment to both fibronectin and polylysinecoated petri dishes induced dephosphorylation of YAP and TAZ and expression of CTGF (Fig. 1G). Altogether, these results indicate that FA formation is not required for cell attachment-induced YAP activation, although, as suggested by following data, FA may play a role in YAP regulation during long-term attachment by organizing cytoskeleton.

Cell attachment also leads to dramatic cytoskeleton reorganization (Mammoto and Ingber 2009). While FA formation was only induced by fibronectin-mediated attachment, cytoskeleton reorganization as well as cytoplasmic translocation of YAP were also seen in polylysine-mediated attachments. Cells attached to fibronectin-coated coverglasses spread and formed cortical actin networks and stress fibers (Fig. 1F). Due to the lack of FAs, cells attached to polylysine-coated material displayed a different F-actin structure, with abundant filopodia at $1 \mathrm{~h}$ after attachment and intricate actin networks in cell body and lamellipodia at $2 \mathrm{~h}$ after attachment. Stress fibers were also occasionally seen (Fig. 1F). In addition, the microtubule cytoskeleton was also established and expanded while cells spread. We did not observe any obvious difference in the microtubule cytoskeleton between cells attached to fibronectin- and polylysine-coated coverglasses, although we saw microtubule extending into filopodia in cells on polylysine (Fig. 1F). These observations raise the possibility that cytoskeleton reorganization may mediate the regulation of YAP by cell attachment.

\section{Actin and microtubule cytoskeletons play important roles in regulation of YAP}

To directly test whether cytoskeleton reorganization plays a role in YAP regulation by cell attachment, we used cytoskeleton-disrupting reagents. Latrunculin B, which disrupts actin cytoskeleton by preventing actin polymerization, strongly blocked attachment-induced 
Zhao et al.

A

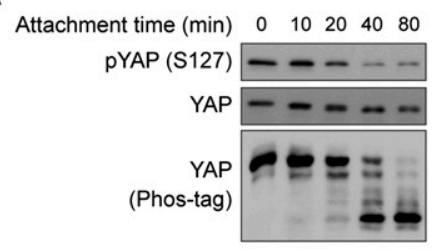

B Lambda PPase - - + +

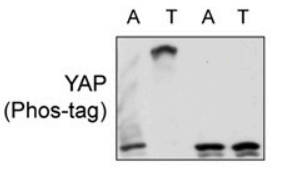

D

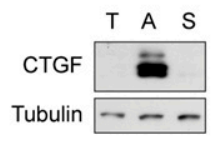

C

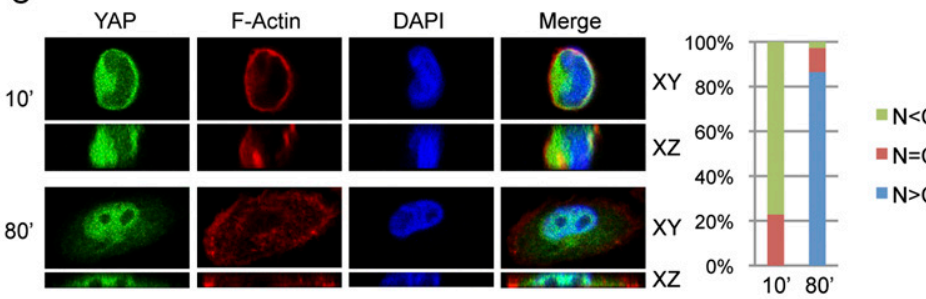

E

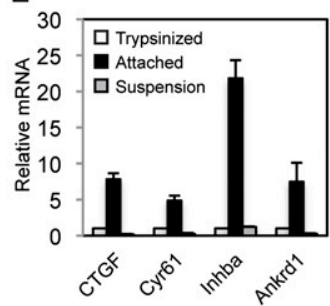

F Fibronectin Poly-lysine Vinculin
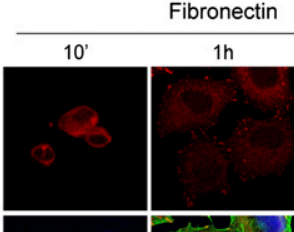

$2 \mathrm{~h}$

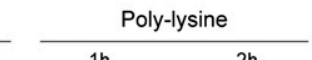

G

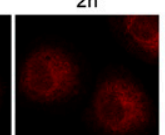

(Phos-tag)

Vinculin
F-actin
DAPI
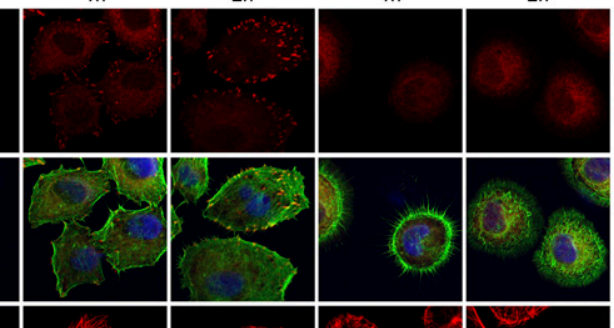

Microtubule

Microtubule
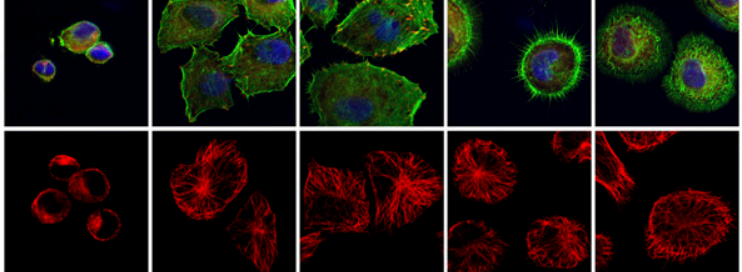

YAP
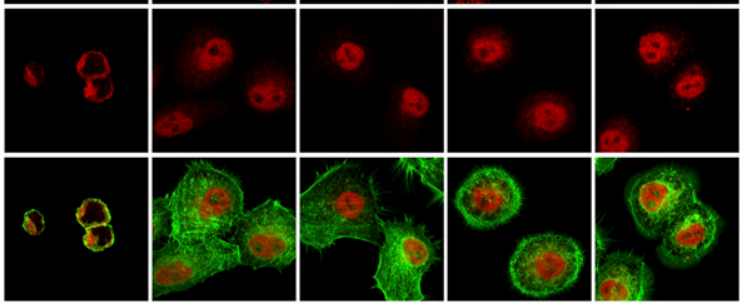

Figure 1. YAP phosphorylation, subcellular localization, and activity are regulated by cell attachment status. $(A)$ Cell attachment induces dephosphorylation of YAP. MCF10A cells were trypsinized and attached onto fibronectin-coated petri dishes for the indicated time. Cells were then lysed, and cell lysates were analyzed by Western blots with the indicated specific antibodies. Phos-tag-containing SDS-PAGE gels were used as indicated. $(B)$ Cell detachment strongly induces YAP phosphorylation. MCF10A cells were trypsinized and attached onto fibronectin-coated petri dishes for $2 \mathrm{~h}$. Attached cells were either directly lysed (A) or trypsinized again before being collected and lysed $(\mathrm{T})$. Aliquots of samples were further treated with $\lambda$ protein phosphatase as indicated. Cell lysates were resolved on Phos-tag-containing SDS-PAGE gels, and anti-YAP antibody was used for Western blot analysis. $(C)$ Cell attachment induces nuclear translocation of YAP. MCF10A cells were trypsinized and attached onto fibronectin-coated coverglasses for the indicated time (in minutes). Cells were then fixed and stained with anti-YAP antibody. Cell boundaries were visualized by staining of F-actin with rhodamine-phalloidin. Both XY views and XZ views from confocal imaging are presented in the left panel. Cells in five random views $(\sim 100$ cells) were quantified for YAP localization and are shown in the right panel. $(D)$ Cell attachment activates CTGF expression. High cell density HeLa cells were trypsinized and lysed $(\mathrm{T})$ or further cultured onto fibronectin-coated petri dishes (A) or onto ultralow attachment plates in suspension (S) for $4 \mathrm{~h}$. Cell lysates were examined by Western blot analysis using the indicated antibodies. (E) Cell attachment activates YAP target gene expression. Experiments were similar to that in $D$. RNA was extracted, and expression of YAP target genes was analyzed by quantitative RT-PCR. $(F)$ Attachment to fibronectin- or polylysine-coated supporting materials similarly induces nuclear translocation of YAP. MCF10A cells were trypsinized and attached onto fibronectin- or polylysine-coated coverglasses for the indicated time in serum-free medium. Cells were then fixed and stained with the indicated antibodies for vinculin, microtubule, or YAP, and with Alexa Fluor 488-conjugated phalloidin for F-actin. Cell nuclei were stained with DAPI. (G) Attachment to fibronectinor polylysine-coated supporting materials similarly activates YAP and TAZ. Experiments were similar to that in $F$. Cells lysates were analyzed by Western blots. Phos-tag-containing SDS-PAGE gels were used as indicated. The asterisk indicates a nonspecific band. 
YAP dephosphorylation (Fig. 2A). A similar effect was also observed when cells were treated with cytochalasin $\mathrm{D}$, another inhibitor that disrupts the actin cytoskeleton by capping filament plus ends (Supplemental Fig. S2A). Consistent with the dispensability of FAs in YAP regulation by cell attachment, inhibitors against signaling molecules in FAs-such as Scr family tyrosine kinase inhibitor PP2, FAK inhibitor PF271, and Rho kinase (ROCK) inhibitor Y27632_-did not significantly inhibit attachmentinduced dephosphorylation of YAP (Supplemental Fig. S2B). Disruption of the actin cytoskeleton abrogates F-actin tension, suggesting a possible role of mechanical force in YAP regulation. However, blebbistatin, which also relieves tension on the actin cytoskeleton by inhibiting myosin II ATPase, did not show an effect similar to latrunculin B (Supplemental Fig. S2A), suggesting that actin-dependent tension is not required for YAP dephosphorylation induced by cell attachment.

The microtubule cytoskeleton is also reorganized during cell attachment. However, disruption of microtubule polymerization by nocodazole or vinblastine as well as induction of microtubule polymerization by taxol did not block attachment-induced YAP dephosphorylation (Supplemental Fig. S2B). Interestingly, pretreatment of cells with nocodazole strongly blocked detachmentinduced YAP phosphorylation (Fig. 2B). This effect was specific to microtubule disruption because concomitant treatment with microtubule-stabilizing reagent taxol neutralized the effect of nocodazole. Again, detachmentinduced YAP phosphorylation was not abolished by PP2, PF271, or blebbistatin treatment (Supplemental Fig. S2C). These data indicate that both actin and microtubule cytoskeletons are involved in YAP regulation by the cell attachment status (Fig. 6D, below). However, they display opposite effects, as F-actin disruption blocks attachment-induced YAP dephosphorylation, whereas microtubule disruption blocks detachment-induced YAP phosphorylation.

We next examined whether cytoskeletons could play a role in YAP regulation in a context other than cell attachment. When cultured at high cell density, YAP phosphorylation is relatively high. In these cells, disruption of microtubule by nocodazole or vinblastine also induced YAP dephosphorylation (Fig. 2C). However, blebbistatin and ROCK inhibitors Y27632 and H1125 did not repress YAP phosphorylation (Supplemental Fig. S2D). In lowdensity cell culture, YAP phosphorylation is at a low level. Disruption of the actin cytoskeleton by latrunculin B or cytochalasin D strongly induced YAP phosphorylation, while relieving tension on the actin cytoskeleton by blebbistatin had a minor effect (Fig. 2D). Actin and microtubule cytoskeletons have extensive physical and functional interactions. Intrigued by the opposite effects of F-actin and microtubule disruption on YAP phosphorylation, we asked whether they were functionally linked in terms of YAP regulation. Interestingly, the dephosphorylation of YAP by disruption of microtubule was largely blocked by codisruption of the actin cytoskeleton (Fig. $2 \mathrm{E}$ ), indicating that these two cytoskeleton systems coordinately regulate YAP phosphorylation.
Consistent with changes in YAP phosphorylation, cytoskeleton integrity also regulates YAP subcellular localization. At low cell density, endogenous YAP mainly localized to the nucleus, while disruption of the actin cytoskeleton by latrunculin B induced YAP cytoplasmic translocation (Fig. 2F). YAP mainly resided in the cytoplasm at high cell confluence. Interestingly, disruption of the microtubule cytoskeleton by nocodazole (Supplemental Fig. S2E) led to nuclear translocation of YAP, which was enhanced by cotreatment with leptomycin $\mathrm{B}$ (LMB), a CRM1-dependent nuclear export inhibitor (Fig. 2F). LMB by itself did not significantly induce translocation of YAP under the condition tested. Nocodazole treatment also induced actin stress fiber formation in some cells (Supplemental Fig. S2E). However, induction of stress fibers by cell-permeable phalloidin did not affect YAP phosphorylation (data not shown), and attachmentinduced YAP dephosphorylation occurred before the formation of stress fibers (Fig. 1F). Thus, the effect of nocodazole on YAP phosphorylation cannot simply be explained by the induction of stress fibers. These data suggest that actin and microtubule cytoskeletons play important roles in the regulation of YAP phosphorylation and localization, and the remodeling of cytoskeleton likely is a key mechanism responsible for regulation of YAP by cell attachment status (Fig. 6D, below).

The Rho family small GTPases play a key role in actin cytoskeleton organization. To determine whether any of the Rho family GTPases was involved in YAP regulation by cell attachment, first we examined whether coexpression of these GTPases modulated YAP phosphorylation. Interestingly, coexpression of either wild-type or constitutively active but not dominant-negative RhoA strongly induced dephosphorylation of YAP (Fig. 2G). Furthermore, coexpression of constitutively active Rac or cdc42, the other two Rho family members, also induced YAP dephosphorylation. The wild-type Rac and cdc42 had only a minor effect (Fig. 2G). To further determine whether any of these small GTPases plays a role in cytoskeleton-mediated regulation of YAP phosphorylation upon cell attachment, we tested cells with Rho or Rac inhibitors. Strikingly, botulinum toxin C3, a specific inhibitor of Rho but not Rac or cdc42 GTPases, completely blocked YAP dephosphorylation induced by cell attachment to both fibronectin and polylysine-coated culture surfaces (Fig. 2H). In contrast, the Rac-specific inhibitor NSC23766 did not affect cell attachmentinduced YAP dephosphorylation. These results suggest that Rho plays a critical role in mediating the effect of cell attachment on YAP phosphorylation, likely through actin cytoskeleton organization, whereas Rac and cdc42 may regulate YAP phosphorylation in another context.

The Hippo pathway is involved in regulation of YAP subcellular localization by the cytoskeleton

As shown above, cell attachment and the cytoskeleton regulate YAP phosphorylation and subcellular localization. We hypothesized that the Hippo pathway might mediate 
Zhao et al.

A

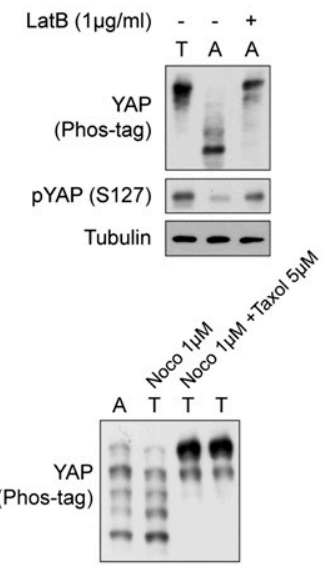

C
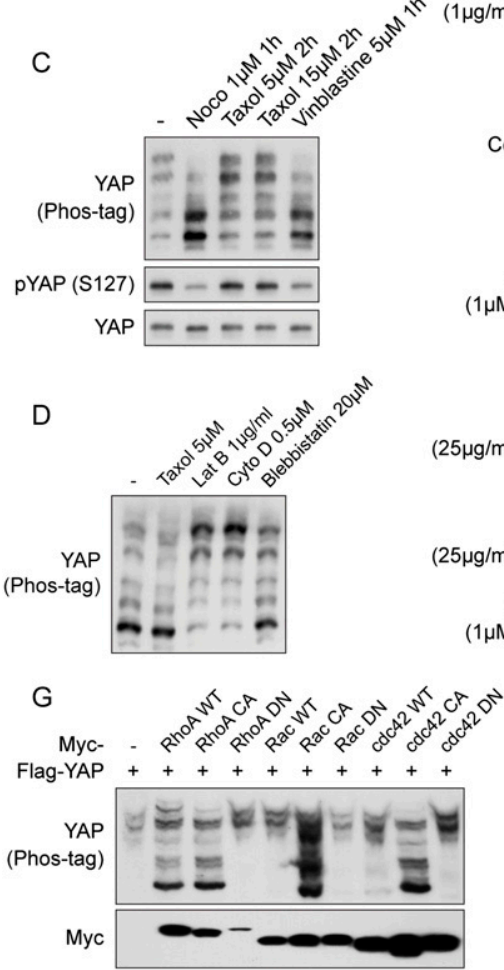

$\mathrm{E}$

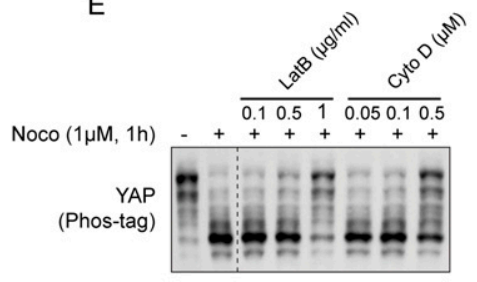

$\mathrm{F}$
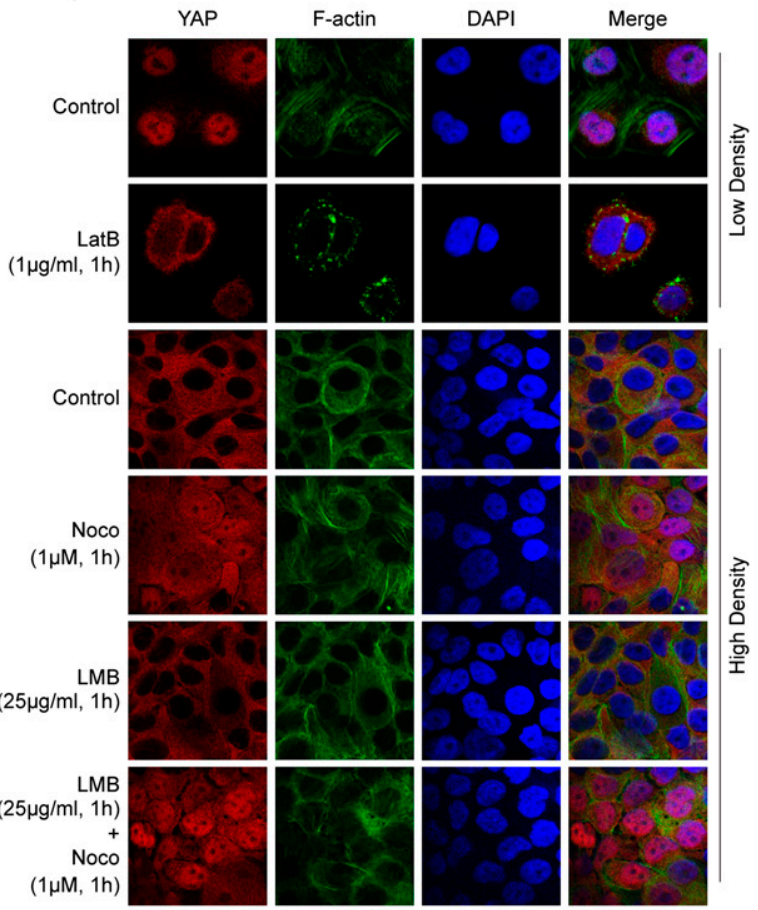

$\mathrm{H}$

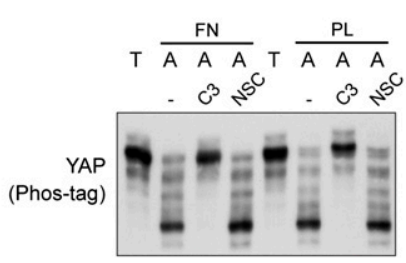

Figure 2. YAP is regulated by the actin and microtubule cytoskeletons. (A) Cell attachment-induced YAP dephosphorylation requires actin polymerization. MCF10A cells were trypsinized (T) and attached onto fibronectin-coated petri dishes (A) for 80 min. Latrunculin $\mathrm{B}$ was added as indicated at the time of plating. Cell lysates were analyzed by Western blots as indicated. $(B)$ Microtubule organization is required for cell detachment-induced YAP phosphorylation. MCF10A cells were trypsinized and attached onto fibronectin-coated petri dishes for $2 \mathrm{~h}$. Cells were either directly lysed (A) or trypsinized again before being collected and lysed (T). Nocodazole (Noco) and taxol were added to cell culture medium $15 \mathrm{~min}$ before and during trypsinization as indicated. $(C)$ Disruption of the microtubule decreases YAP phosphorylation. MCF10A cells cultured to $80 \%$ confluence were treated with the indicated chemicals in culture medium. Cells were then lysed and analyzed by Western blots. $(D)$ Disruption of the actin cytoskeleton induces YAP phosphorylation. MCF10A cells were trypsinized and attached onto fibronectin-coated petri dishes for $2 \mathrm{~h}$, followed by addition of the indicated reagents, and were further cultured for another hour before being harvested for Western blot analysis. $(E)$ F-actin is required for YAP dephosphorylation induced by microtubule depolymerization. MCF10A cells were cultured to confluence and treated with inhibitors in cultured medium as indicated. Cell lysates were resolved on Phos-tag-containing SDS-PAGE gels, and anti-YAP antibody was used for Western blotting. Data were cropped from the same exposure of the same blot. $(F)$ The actin and microtubule cytoskeletons regulate YAP subcellular localization. MCF10A cells were cultured onto fibronectin-coated coverglasses at low or high cell density. Cells were treated with the indicated inhibitors before fixation. Samples were then stained with anti-YAP antibody for endogenous YAP, Alexa Fluor 488-conjugated phalloidin for F-actin, and DAPI for cell nuclei. $(G)$ Rho family small GTPases induce YAP dephosphorylation. HEK293 cells were cotransfected with the indicated plasmids. Cells were then lysed and analyzed by Western blots. $(H)$ Rho mediates cell attachment-induced YAP dephosphorylation. MCF10A cells were trypsinized and then attached to fibronectin- or polylysinecoated petri dishes for $2 \mathrm{~h}$ in the presence of Rho inhibitor C3 $(2 \mu \mathrm{g} / \mathrm{mL})$ or Rac inhibitor NSC23766 (100 $\mu$ M). Cell lysates were resolved on Phos-tag-containing SDS-PAGE gels, and anti-YAP antibody was used for Western blotting. 
these effects. To test this hypothesis, we first compared the effect of cytoskeleton disruption on the localization of YAP wild type and 5SA, a YAP mutant with all five Lats $1 / 2$ serine phosphorylation sites mutated to alanine, which is unresponsive to the Hippo pathway. Ectopically expressed YAP wild-type protein mainly adopted cytoplasmic localization even with LMB treatment when cells were confluent (Fig. 3A). Similar to endogenous protein, treatment of cells with nocodazole induced nuclear accumulation of wild-type YAP (Fig. 3A). However, the YAP-5SA mutant mainly localized to the nucleus regardless of microtubule integrity, suggesting that the microtubule regulates YAP localization via the Lats $1 / 2$ phosphorylation sites on YAP. At low cell density, ectopically expressed YAP showed prominent nuclear localization with some perinuclear localization. Disruption of the actin cytoskeleton by latrunculin B decreased nuclear YAP and increased cytoplasmic YAP (Fig. 3B). However, YAP-5SA did not show cytoplasmic translocation in response to latrunculin $\mathrm{B}$. These observations suggest that YAP phosphorylation by the Hippo pathway is important for regulation of YAP localization by the cytoskeleton.

\section{Cell attachment and the cytoskeleton regulate YAP by modulating Lats1/2 activity}

We then directly tested whether attenuation of Hippo pathway kinases would affect cell detachment-induced YAP phosphorylation. In control HeLa cells, YAP was
A
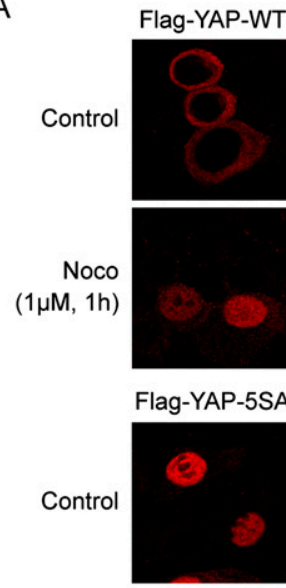

Noco $(1 \mu \mathrm{M}, 1 \mathrm{~h})$
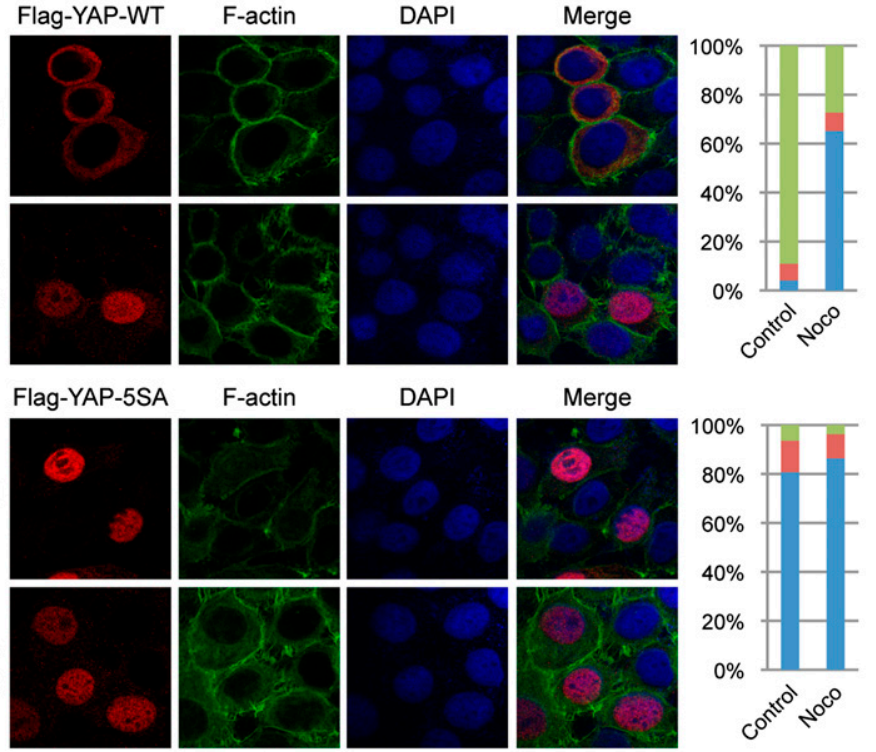

$=\mathrm{N}<\mathrm{C}$

$=\mathrm{N}=\mathrm{C}$

$=\mathrm{N}>\mathrm{C}$
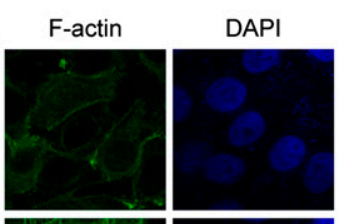

Merge
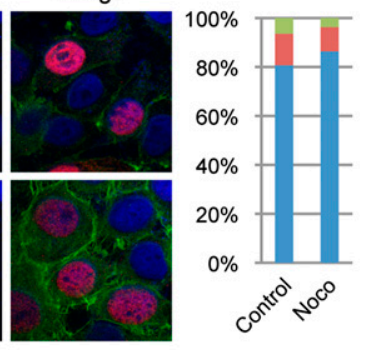

B
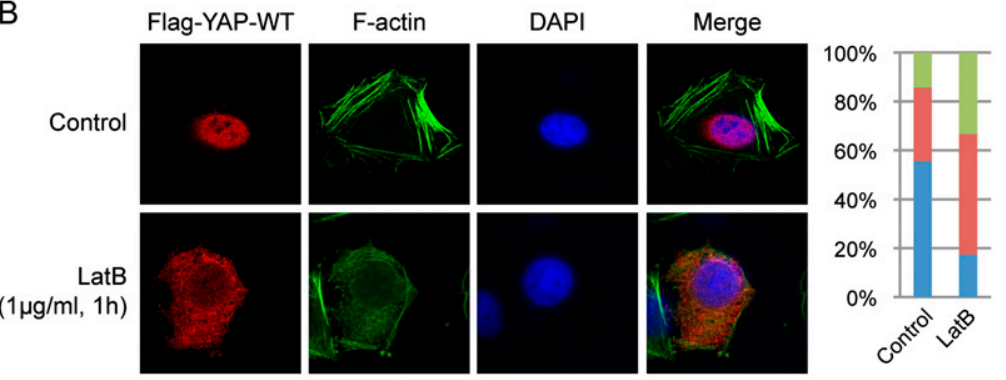

$=\mathrm{N}<\mathrm{C}$

$=\mathrm{N}=\mathrm{C}$

$=\mathrm{N}>\mathrm{C}$
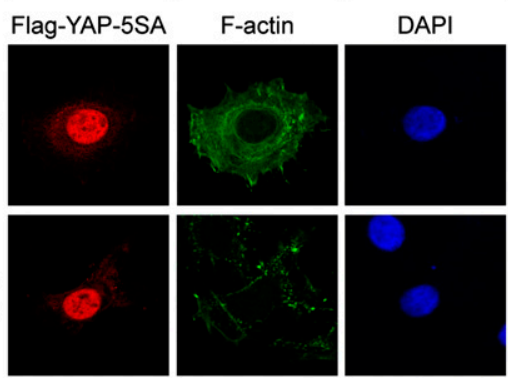

Control

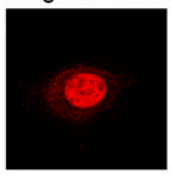

LatB

$(1 \mu \mathrm{g} / \mathrm{ml}, 1 \mathrm{~h})$

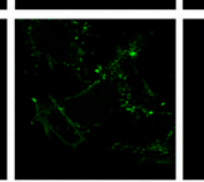

Figure 3. The cytoskeleton regulates YAP subcellular localization through the Hippo pathway phosphorylation sites. (A) Subcellular localization of YAP wild type but not the 5SA mutant is regulated by microtubule cytoskeleton integrity. HeLa cells were transfected with Flag-YAP wild type or 5SA mutant and then cultured onto fibronectincoated coverglasses at high cell density. Cells were treated with $25 \mu \mathrm{g} / \mathrm{mL}$ LMB $3 \mathrm{~h}$ before fixation. In the last hour, nocodazole was added as indicated. Cells were stained with anti-Flag tag antibody for YAP, Alexa Fluor 488-conjugated phalloidin for F-actin, and DAPI for cell nuclei. Cells in five random views $(\sim 100$ cells) were quantified for YAP localization and are shown in the right panels. (B) Localization of YAP wild type but not the 5SA mutant is responsive to actin cytoskeleton integrity. HeLa cells were transfected as in $A$ and then cultured onto fibronectin-coated coverglasses at low cell density. Cells were treated with latrunculin B $1 \mathrm{~h}$ before fixation as indicated. Cells were stained with anti-Flag tag antibody for YAP, Alexa Fluor 488-conjugated phalloidin for F-actin, and DAPI for cell nuclei. Cells in five random views $(\sim 100$ cells) were quantified for YAP localization and are shown in the right panels. 
phosphorylated upon cell trypsinization and was dephosphorylated when cells were attached. Further retrypsinization led to YAP phosphorylation again (Fig. 4A). However, when Lats1/2 were down-regulated by siRNAs (Supplemental Fig. S3A), the YAP phosphorylation level was much lower regardless of cell attachment status, suggesting that Lats1/2 were the kinases responsible for YAP phosphorylation in both attachment and detachment
(Fig. 4A). Mst1/2 are known upstream kinases activating Lats1/2 (Chan et al. 2005). Although knockdown of Mst1/2 by siRNAs did not affect basal YAP phosphorylation in HeLa cells, it did attenuate YAP phosphorylation when cells were detached, and the effect lasted to the next attachment step (Fig. 4A). This result suggests that Mst1/2 contribute to YAP phosphorylation under detached condition in this cell type. These observations indicate
A

siRNA Control Lats1+Lats2 Mst1+Mst2

- $T$ A reT - T A reT - T A reT

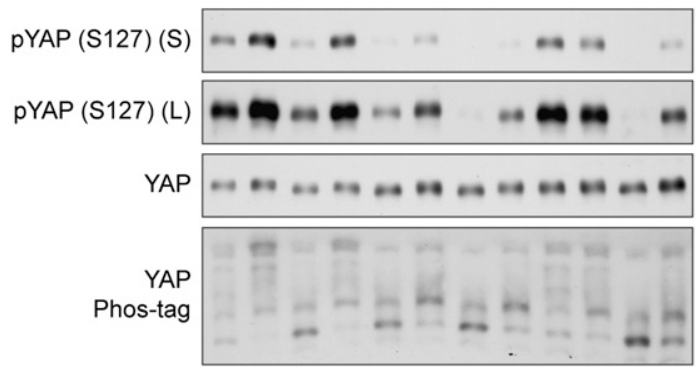

C

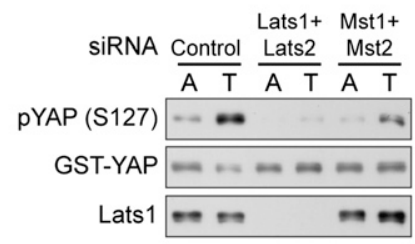

$\mathrm{F}$

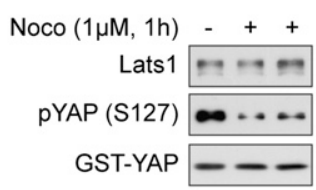

$\mathrm{D}$

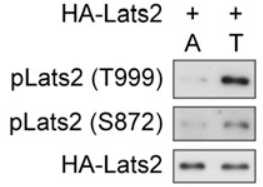

G

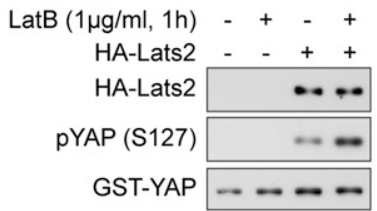

\section{B}

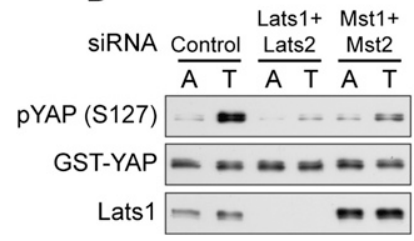

E

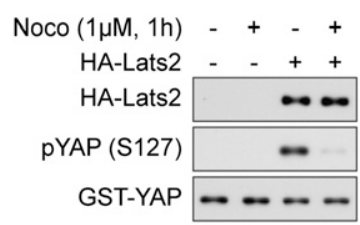

$\mathrm{H}$

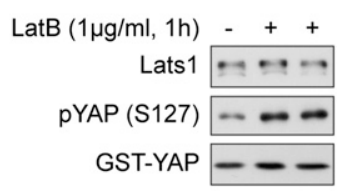

Figure 4. Cell attachment and cytoskeleton remodeling regulate Lats $1 / 2$ kinase activity. $(A)$ The Hippo pathway kinases are crucial in cell detachment-induced YAP phosphorylation. HeLa cells were transfected with the indicated siRNAs twice with a 24-h interval. Cells either were directly lysed or went through the following treatment: trypsinized ( $T$ ), trypsinized and then attached to fibronectin-coated petri dishes for $2 \mathrm{~h}(\mathrm{~A})$, or further trypsinized after attachment (reT). Samples were analyzed by Western blots as indicated. S and $\mathrm{L}$ indicate short and long exposures, respectively. $(B)$ A YAP kinase is activated by cell detachment. HeLa cells were transfected with the indicated siRNAs twice with a 24-h interval. Cells were replated onto fibronectin-coated petri dishes for $3 \mathrm{~h}$ and then directly lysed (A) or lysed following trypsinization (T). Cell lysates were then incubated with GST-YAP immobilized on glutathione resin. The resins were then washed and subjected to in vitro kinase assays. The products were analyzed by Western blots as indicated. $(C)$ Lats 1 is activated by cell detachment. Cell lysates were prepared as in $B$. Lats 1 immunoprecipitated from the cell lysates was subjected to in vitro kinase assays using GST-YAP purified from Escherichia coli as a substrate. The products were analyzed by Western blotting as indicated. $(D)$ Cell detachment increases Lats2 phosphorylation. HeLa cells transfected with HA-Lats2 were replated onto fibronectincoated petri dishes for $3 \mathrm{~h}$ and then directly lysed (A) or lysed following trypsinization (T). HA-Lats 2 was immunoprecipitated with antiHA antibody and then subjected to Western blot analysis as indicated. $(E)$ Lats2 is inhibited by microtubule disruption. HeLa cells transfected with HA-Lats2 and cultured at high cell density were treated as indicated. Untransfected cells were used as negative control. HA-Lats2 immunoprecipitated with anti-HA antibody was subjected to in vitro kinase assay using GST-YAP as a substrate. The products were analyzed by Western blotting as indicated. $(F)$ Endogenous Lats 1 is inhibited by disruption of the microtubule. Experiments were similar to that in $E$ except that endogenous Lats1 was immunoprecipitated from untransfected HeLa cells. $(G)$ Lats2 is activated by actin depolymerization. Experiments were similar to that in $E$ except that HeLa cells were newly plated on fibronectincoated petri dishes and treated with latrunculin B as indicated. $(H)$ Endogenous Lats1 is activated by actin depolymerization. Experiments were similar to those in $G$ except that endogenous Lats1 was immunoprecipitated from untransfected HeLa cells. 
that the Hippo pathway regulates YAP phosphorylation in response to cell attachment status.

To test whether a YAP kinase was activated during cell detachment, we assayed YAP phosphorylation in vitro. To do so, we incubated immobilized GST-YAP with cell lysates to pull down its upstream kinase. The activity of this captured kinase could be examined by in vitro kinase assay. Interestingly, the YAP kinase captured by GSTYAP pull-down from lysates of trypsinized HeLa cells showed markedly elevated activity compared with that from lysates of attached cells (Fig. 4B), indicating that a YAP kinase is activated by detachment. Interestingly, using a Lats1-specific antibody, we detected Lats1 in the pull-down products of GST-YAP (Fig. 4B). Furthermore, knockdown of Lats1/2 or Mst1/2 (Supplemental Fig. S3B) largely decreased the activation of this YAP kinase by cell detachment, suggesting that this kinase might be Lats1/ 2. Next, we immunoprecipitated endogenous Lats1 from attached or trypsinized HeLa cells and directly examined its activity in vitro. Lats1 was strongly activated by cell detachment, and both Lats1/2 and Mst1/2 knockdown decreased Lats1 activity (Fig. 4C). Lats1/2 are activated by phosphorylation of the activation loop (S909/872 for Lats1 and Lats2) and the hydrophobic motif (T1079/999 for Lats1 and Lats2) (Chan et al. 2005). Using two phospho-specific antibodies, we detected a significant increase of phosphorylation of ectopically expressed Lats2 in trypsinized cells (Fig. 4D), further supporting the activation of Lats 2 by cell detachment. The specificity of these antibodies was confirmed for ectopically expressed Lats2 (Supplemental Fig. S3C), although we had difficulty detecting endogenous pLats $1 / 2$ using these antibodies in the conditions we tested (data not shown). Therefore, our results unequivocally demonstrate for the first time that cell detachment activates the Hippo pathway kinases Lats $1 / 2$.

If cell attachment status regulates YAP phosphorylation through cytoskeleton remodeling, disruption of cytoskeletons should also affect Lats $1 / 2$ activity. Indeed, disruption of the microtubule cytoskeleton by nocodazole significantly decreased the activity of both ectopically expressed Lats2 and endogenous Lats1 (Fig. 4E,F). In contrast, disruption of the actin cytoskeleton by latrunculin B activated both ectopically expressed Lats2 and endogenous Lats1 (Fig. 4G,H). The above experiments provide direct evidence that the Hippo pathway mediates regulation of YAP phosphorylation and localization by cell detachment and cytoskeleton integrity.

\section{Mst1/2 activity is not regulated by cell attachment}

Mst1/2 are upstream activating kinases of Lats $1 / 2$ in the Hippo pathway. To determine whether Mst $1 / 2$ activation might be responsible for Lats $1 / 2$ activation by cell detachment, we examined Mst1/2 kinase activity in vitro toward Mob, which was reported to be a direct substrate of Mst1/2 (Praskova et al. 2008). Surprisingly, Mst2 immunoprecipitated from transfected HeLa cells in attached or trypsinized conditions showed similar activity, as indicated by comparable Mst2 autophosphorylation and
Mob phosphorylation shown by ${ }^{32} \mathrm{P}$-ATP incorporation (Supplemental Fig. S4A). To further confirm this observation, we immunoprecipitated endogenous Mst1 from attached or detached MCF10A cells and found that endogenous Mst1 activity remained the same in the two conditions, although endogenous YAP phosphorylation was clearly stimulated by detachment (Supplemental Fig. S4B). Mst $1 / 2$ are activated by phosphorylation on the activation loop. Using an antibody that specifically recognizes the phosphorylated and hence active Mst1/2, we found that the activating phosphorylation of Mst2 was not affected by cell attachment status (Supplemental Fig. S4C). Consistently, endogenous Mob phosphorylation was not regulated by cell attachment, although it was activated by hydrogen peroxide treatment as previously reported (Supplemental Fig. S4D). These data suggest that Mst $1 / 2$ kinase activity is not regulated by cell attachment.

We further examined YAP phosphorylation in Mst1 and Mts2 double-knockout mouse embryonic fibroblast (MEF) cells and found that YAP phosphorylation was still efficiently regulated by cell attachment (Supplemental Fig. S4E). However, in HeLa cells, Mst1/2 appeared to play a role in detachment-induced YAP phosphorylation (Fig. 4A-C). Thus, the requirement of Mst1/2 in Lats1/2 and YAP regulation by cell attachment seems to be cell type-dependent, which is consistent with previous reports in other context (Zhou et al. 2009). In cells such as MEFs, Mst $1 / 2$ is not required, and in other cells such as HeLa cells, Mst1/2 are involved, although their kinase activity is not regulated by cell attachment. We also examined the possibility that cell attachment regulates the interaction among Hippo pathway core components Mst1/2, Sav, Lats1/2, and Mob. However, by coimmunoprecipitation experiments, we did not observe significant changes in Mst2-Sav interaction and Lats2-Mob interaction (Supplemental Fig. S5A) or alteration of the four-component complex formation (Supplemental Fig. S5B). Based on current observations, we propose that cell attachment regulates the Hippo pathway downstream from Mst1/2 and upstream of Lats1/2.

\section{YAP inhibits anoikis of mammary epithelial cells}

Accumulating evidence supports an oncogenic role of YAP and a tumor suppressor function of the Hippo pathway (Overholtzer et al. 2006; Zender et al. 2006; Zhao et al. 2007; Steinhardt et al. 2008; Xu et al. 2009; Zhou et al. 2009; Lee et al. 2010; Lu et al. 2010; Pan 2010; Song et al. 2010). Therefore, the regulation of YAP by cell attachment status suggests a possible role of the Hippo pathway in anoikis, which is initiated by detachment and represents a barrier for tumor cell survival in the bloodstream and metastasis (Chiarugi and Giannoni 2008). We hypothesize that activation of the Hippo pathway and subsequent inhibition of YAP represent a mechanism to activate anoikis and inhibit metastasis. As shown in Figure 5A and as previously reported (Zhao et al. 2008), active YAP could induce MCF10A cell foci formation in soft agar, suggesting that YAP not only promotes proliferation, but also inhibits anoikis. To directly assay the effect of YAP on 
Zhao et al.

A

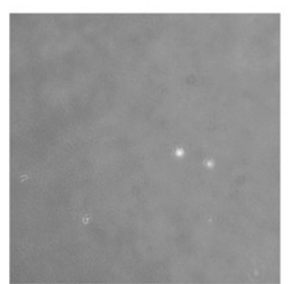

Vector

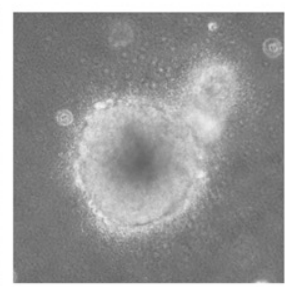

YAP-5SA

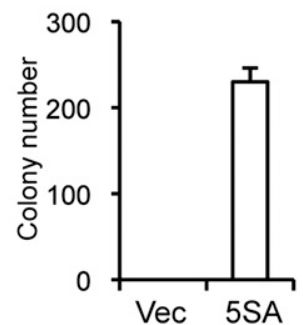

B

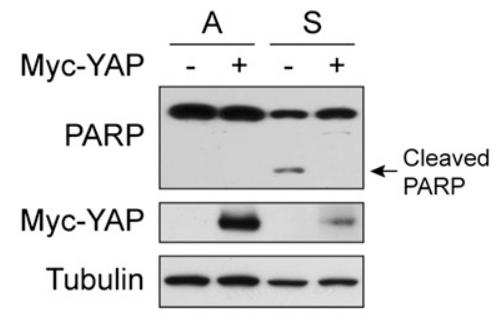

C
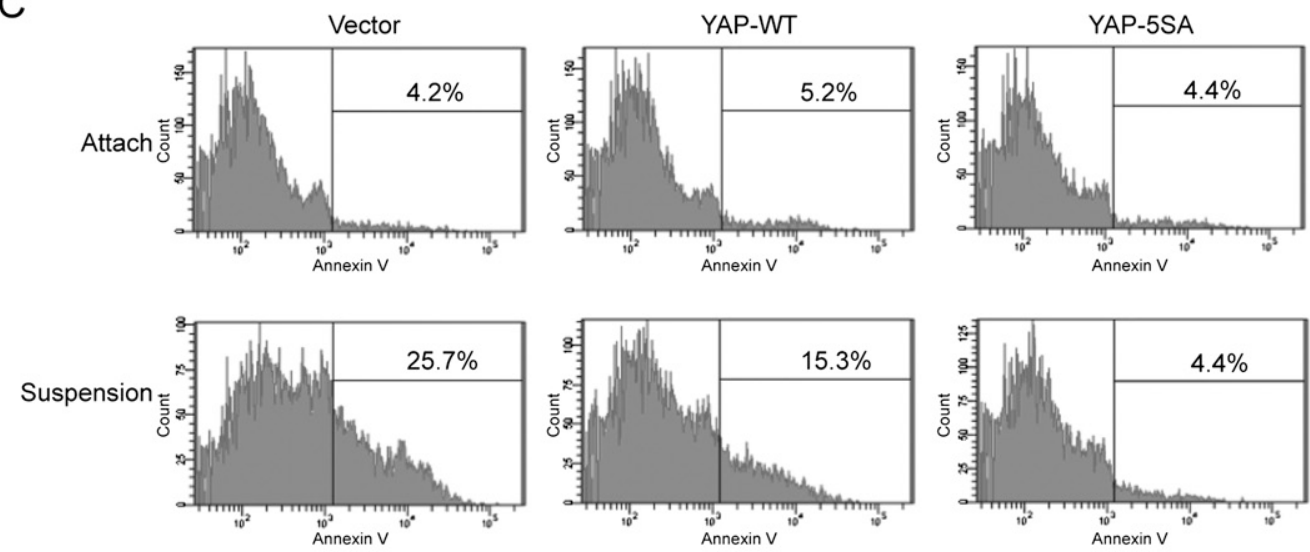

$\mathrm{D}$
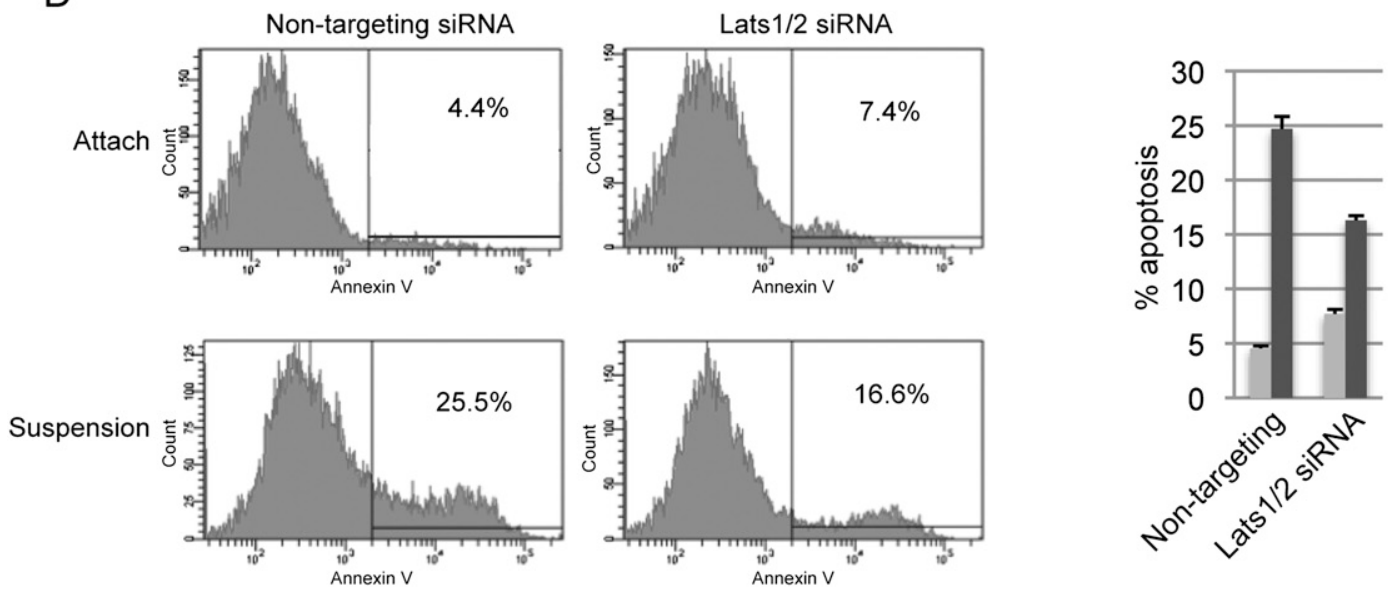

Attach

- Suspension

Figure 5. YAP inhibits detachment-induced anoikis. (A) Active YAP induces anchorage-independent growth in MCF10A cells. MCF10A cells infected with control vector or YAP-5SA were cultured in soft agar for $16 \mathrm{~d}$ before pictures were taken. Colony number was counted and is shown in the right panel. $(B)$ YAP inhibits suspension-induced PARP cleavage. Control or YAP-expressing HMLE cells were cultured on tissue culture plates (A) or ultralow attachment plates in suspension (S) for $72 \mathrm{~h}$. Cells were then harvested and analyzed by Western blotting with specific antibodies. (C) YAP inhibits anoikis. HMLE cells with control vector or ectopic expression of YAP wild type or 5SA mutant were cultured on tissue culture plates (Attach) or ultralow attachment plates in suspension for $72 \mathrm{~h}$. Cells were then collected and stained with PE Annexin V and analyzed by FACS. Experiments were done in duplicate. $(D)$ Knockdown of Lats1/2 inhibits anoikis in HMLE cells. HMLE cells were transfected with control siRNA or siRNAs targeting Lats1 and Lats2. Cells were then cultured on tissue culture plates (Attach) or ultralow attachment plates in suspension. After $72 \mathrm{~h}$, cells were collected and stained with PE Annexin V and analyzed by FACS. Apoptosis rates were quantified from two independent experiments and are shown in the right panel. 
anoikis, we did suspension culture of MCF10A and another noncancerous mammary epithelial cell line, HMLE, on ultralow attachment plates. Although our MCF10A cells did not show significant anoikis up to $3 \mathrm{~d}$ in suspension culture, possibility due to compensation of the attachment signal by cell aggregation, HMLE did go through anoikis, as indicated by PARP cleavage, a wellestablished apoptosis marker (Fig. 5B). Interestingly, PARP cleavage induced by suspension culture was blocked by ectopic expression of YAP (Fig. 5B). To further confirm the role of YAP in inhibiting anoikis, we stained cells from attachment or suspension culture with Annexin V, an apoptosis marker. While only $4 \%$ of cells in attached culture show positive staining of Annexin V, $25 \%$ of cells in suspension culture were Annexin V positive, indicating induction of anoikis (Fig. 5C). Expression of wild-type YAP reduced anoikis by half, and expression of active YAP-5SA completely blocked anoikis such that the apoptosis rate was similar to that observed in attached cells (Fig. 5C). These data support a critical inhibitory role of YAP in anoikis and the necessity of YAP inactivation in order for anoikis to occur.

YAP is known to induce epithelial-mesenchymal transition (EMT) (Overholtzer et al. 2006; Zhao et al. 2008), which has been shown to repress anoikis in mammary epithelial cells (Onder et al. 2008). Indeed, we observed that expression of wild-type or active YAP induced a gradual loss of E-cadherin and induction of $\mathrm{N}$-cadherin in HMLE cells even in suspension (Supplemental Fig. S6A). Furthermore, we also observed an induction of Erk phosphorylation by YAP specifically in suspension cells (Supplemental Fig. S6A), indicating activation of the classical MAP kinase survival pathway by YAP. A possible mechanism for Erk activation by YAP is that many growth factors are induced by YAP (Zhao et al. 2007; Zhang et al. 2009). Therefore, YAP-induced EMT and Erk activation may contribute to the function of YAP in inhibiting anoikis.

If the Hippo pathway is indeed involved in anoikis, one may speculate that Lats $1 / 2$ would be important for anoikis to occur in untransformed cells. To test this hypothesis, we used siRNAs targeting Lats1 and Lats2 to downregulate their expression in HMLE cells (Supplemental Fig. S6B). Indeed, knockdown of Lats $1 / 2$ attenuated anoikis of HMLE cells by $>50 \%$ (Fig. 5D), a level similar to that induced by ectopic expression of wild-type YAP. Thus, not only YAP overexpression could prevent anoikis, but also the endogenous Hippo pathway is important for anoikis of untransformed cells.

\section{Inactivation of the Hippo pathway contributes to anoikis evasion and correlates with cancer metastasis}

To further investigate the role of the Hippo pathway in anoikis in the context of cancer metastasis, we analyzed anoikis of several prostate cell lines that have different tumorigenic and metastatic potentials. As we expected, the noncancerous prostate cell line RWPE showed significant anoikis when cultured in suspension (Fig. 6A), while the metastatic prostate cancer cell line PC-3 only showed minor anoikis (Supplemental Fig. S6C), and DU 145, a less-metastatic prostate cancer cell line, showed intermediate anoikis (Supplemental Fig. S6D). More importantly, similar to that in HMLE cells, knockdown of Lats1/2 in RWPE cells (Supplemental Fig. S6B) significantly reduced anoikis (Fig. 6A), suggesting a cell typeindependent role of the Hippo pathway in regulating anoikis. Since YAP activation would evade anoikis, we asked whether YAP activity contributed to the lack of anoikis in these anoikis-resistant prostate cancer cells. We used siRNA to knock down both YAP and TAZ, a YAP paralog. However, we did not see a significant increase of anoikis in PC-3 and DU 145 upon YAP/TAZ knockdown (Supplemental Fig. S6C,D). Further analysis indicated that YAP could be phosphorylated in these cells upon cell detachment (data not shown), suggesting that anoikis deficiency in these cancer cells is likely due to an abnormality other than the Hippo pathway.

We reasoned that cancer cells with an abnormal Hippo pathway might rely on YAP/TAZ to suppress anoikis. One such cancer cell line is ACHN, a renal carcinoma cell line that has elevated YAP activity due to Sav mutation (Tapon et al. 2002). Notably, knockdown of YAP and TAZ (Supplemental Fig. S6B) potently promoted anoikis in this cell line (Fig. 6B). Similarly, knockdown of YAP and TAZ also increased anoikis of SF268 (Supplemental Fig. S6E), a glioblastoma cell line with an abnormally high level of YAP expression (K-L Guan and I Lian, unpubl.). These data further support that abnormality of the Hippo pathway could lead to evasion of anoikis, and therapeutics targeting YAP might be an attractive approach to induce anoikis and prevent metastasis of cancers with an aberrant Hippo pathway.

Although accumulating evidence strongly supports a tumor suppressor role of the Hippo pathway and an oncogenic role of YAP in human cancer, there has been no clinical evidence linking the Hippo pathway to tumor metastasis. We asked whether Lats1/2 inhibition might be clinically relevant to human cancer metastasis. To this end, we analyzed Lats1 and Lats2 expression in a cohort of 132 clinically localized prostate cancer, 18 metastatic prostate cancer, and 29 benign adjacent prostate tissues (Taylor et al. 2010). Our analysis revealed significant down-regulation of both Lats1 $(P=0.01)$ and Lats2 $(P=0.0005)$ mRNA levels in metastatic prostate cancer when compared with benign or clinically localized cancer tissues (Fig. 6C). Considering the functional redundancy of Lats1 and Lats2, the total mRNA level of these two genes might be more relevant in terms of YAP phosphorylation level and clinical outcome. We found that the total mRNA level of Lats1 and Lats2 is also significantly down-regulated in metastatic prostate cancer $(P=0.0002)$ (Fig. 6C). Similarly, remarkable downregulation of Lats1 $(P=0.01)$ and Lats2 $(P=3 \mathrm{E}-06)$ was confirmed in an independent cohort of primary tissues consisting of $\sim 30$ adjacent benign tissues and 80 localized and 40 metastatic prostate cancer tissues (Supplemental Fig. S6F). Thus, we report for the first time that down-regulation of the key Hippo pathway kinases Lats $1 / 2$ correlates with metastasis of certain human cancers, such as prostate cancer. Lats1/2 may suppress tumor metastasis by mediating YAP inhibition and anoikis induction. 
Zhao et al.

A
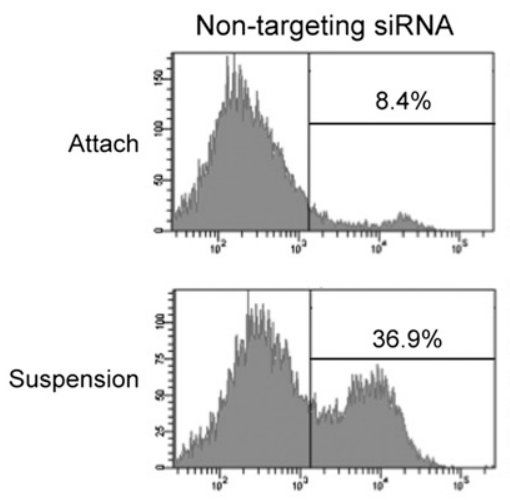

C
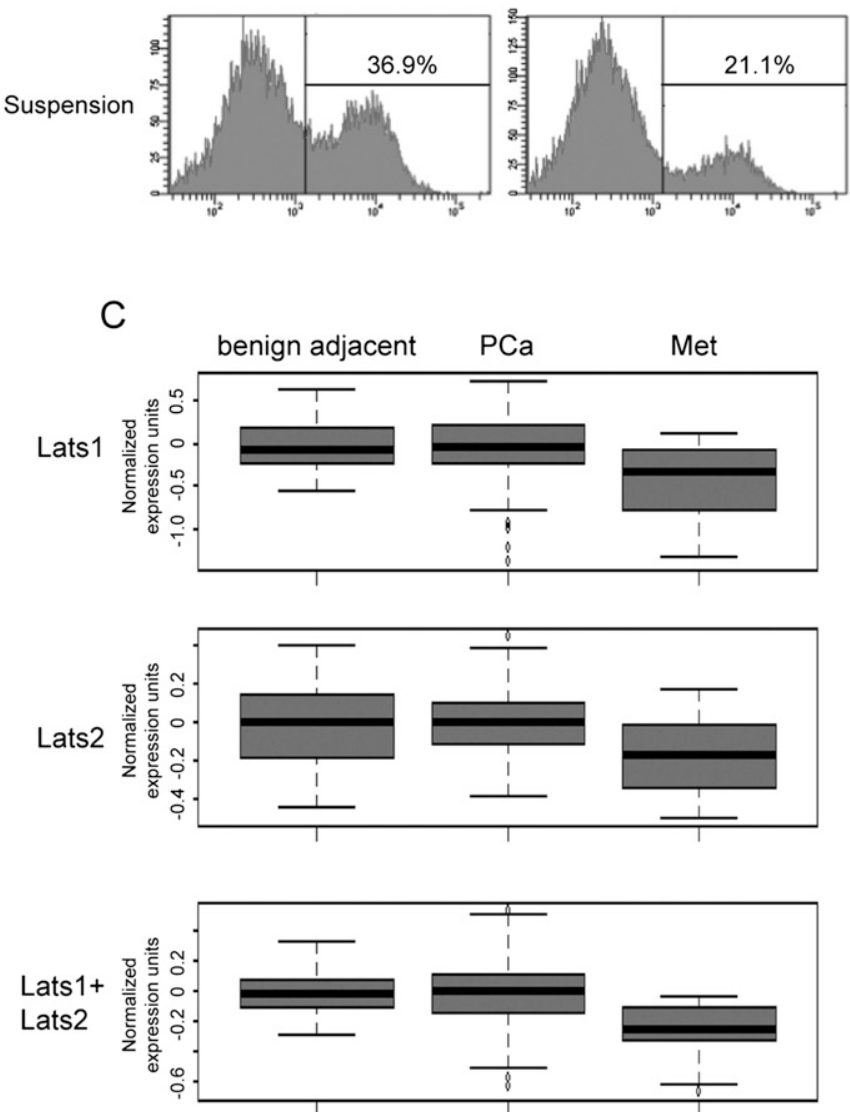

B
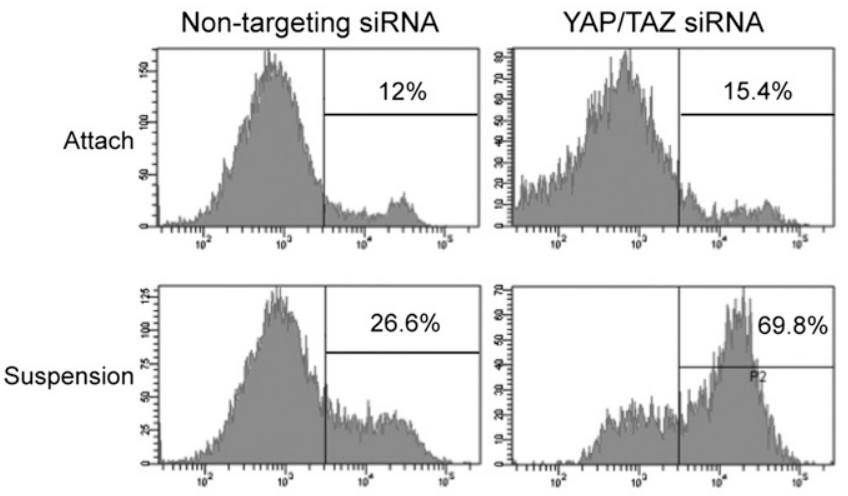

D

$P$ values

0.01

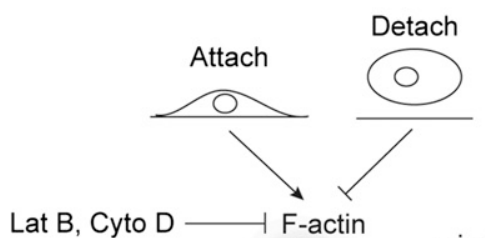

nocodazole

0.0002

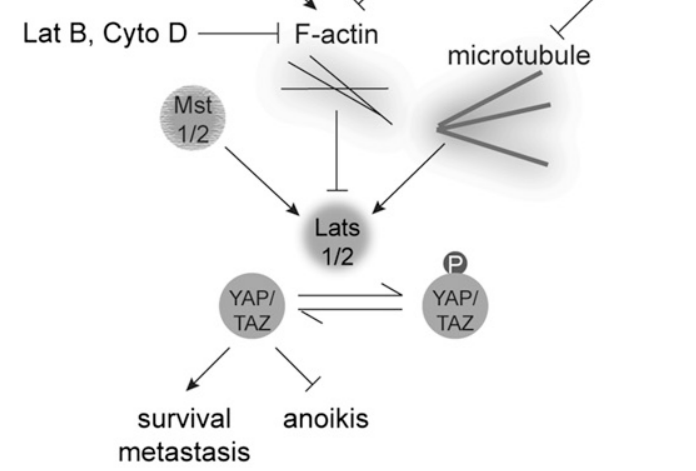

Figure 6. Inactivation of the Hippo pathway contributes to anoikis evasion and correlates with cancer metastasis. (A) Lats $1 / 2$ knockdown impairs anoikis in RWPE cells. RWPE cells were transfected with control siRNA or siRNAs targeting Lats1 and Lats2. Cells were then cultured on tissue culture plates (Attach) or ultralow attachment plates in suspension. After $72 \mathrm{~h}$, cells were collected and stained with PE Annexin V and analyzed by FACS. Experiments were done in duplicate. $(B)$ YAP/TAZ knockdown restores anoikis in ACHN cells. Experiments were similar to that in $A$ except that siRNAs against YAP and TAZ as well as ACHN cells were used. Experiments were done in duplicate. $(C)$ Lats1/2 down-regulation correlates with prostate cancer metastasis. Box plots showing the expression value of Lats1 and Lats2 in benign adjacent, localized $(\mathrm{PCa})$, and metastatic (Met) prostate tumors. $P$-value measures the statistical difference between metastatic prostate cancer when compared with benign tissue and localized prostate cancer. $(D)$ A model of the Hippo pathway mediating the cell attachment signal to anoikis and cancer metastasis.

\section{Discussion}

Despite rapid progress in the field, upstream signals for Hippo pathway regulation remain poorly understood. Here we report that cell-ECM contact regulates Hippo pathway activity through cytoskeleton organization (Fig. 6D). Consistently, two recent studies in Drosophila provide in vivo evidence that induction of extra F-actin network formation induces overgrowth through modulation of the Hippo pathway activity (Fernandez et al. 2011; Sansores-Garcia et al. 2011). In addition, the overgrowth caused by extra
F-actin in Drosophila was suppressed efficiently by Wts overexpression but only mildly by Hpo or Ex overexpression, even though overexpression of Ex or Hpo had stronger effects on growth inhibition than Wts overexpression in wild-type Drosophila (Sansores-Garcia et al. 2011). These observations are consistent with our biochemical data that cell attachment and the cytoskeleton impinge into the Hippo pathway downstream from Mst1/2 and upstream of Lats $1 / 2$.

A recent study suggested that tension on actin stress fiber might promote nuclear localization of YAP and TAZ 
in a Hippo pathway-independent manner (Dupont et al. 2011). However, another report suggested that cell morphology regulates YAP in a phosphorylation-dependent manner (Wada et al. 2011). In the context of cell attachment, we provide compelling evidence that YAP nuclear localization is promoted via inhibition of Lats1/2 kinases through cytoskeleton reorganization. The small GTPase Rho is involved in this regulation. Contrary to previous reports, such regulation does not seem to rely on ROCK activation, contractile actin-myosin filaments formation, or actin tension, although in cells already attached, actin tension does seem to moderately affect YAP phosphorylation. One possibility is that mDia-induced actin polymerization plays a role in Lats $1 / 2$ inhibition downstream from Rho activation. Such speculation is supported by observations in Drosophila (Sansores-Garcia et al. 2011). We recently reported that the angiomotin family proteins could mediate YAP localization to tight junctions and the actin cytoskeleton and promote YAP phosphorylation (Zhao et al. 2011). Whether Lats $1 / 2$ activation induced by cell detachment or cytoskeleton remodeling synergizes with angiomotin-mediated YAP regulation awaits further investigation. Several recent studies provided evidence that disruption of the E-cadherin-catenin complex at the cell-cell junction also leads to activation of YAP (Kim et al. 2011; Schlegelmilch et al. 2011; Silvis et al. 2011). E-cadherin-catenin links cell adhesion to the cytoskeleton structures. It is therefore worthy to speculate that the Lats $1 / 2$ kinases in the Hippo pathway may mediate signals from cell adhesion and the cytoskeleton to cell proliferation and apoptosis, and thus tissue homeostasis.

Besides F-actin, we also demonstrated that the microtubule cytoskeleton plays important roles in stimulating Lats $1 / 2$ kinase activity and YAP phosphorylation. Notably, anti-microtubule agents such as taxol are widely used in cancer treatment. Since disruption of the microtubule inhibits the Hippo pathway and activates YAP, it is conceivable that stabilization of the microtubule by taxol may have a signaling role in YAP inhibition and thus contribute to the anti-tumor effect of the drug besides the well-established function of taxol to interfere with cell cycle progression. Moreover, elevated levels of TAZ, the YAP paralog, were found to be responsible for taxol resistance in breast cancer (Lai et al. 2011). Thus, the connection between the microtubule and the Hippo pathway revealed in this study may have implications in cancer treatment and drug resistance.

How cell-ECM contact regulates cell physiology is a basic cell biology question. While the regulation of cell morphology and cell motility by cell-ECM interaction is relative clear, its regulation of cell proliferation and anoikis is not completely understood. Current studies of anoikis initiation focus on FA-originated signals (Chiarugi and Giannoni 2008). In this study, we show that cell detachment activates the Hippo tumor suppressor pathway, leading to inhibition of the YAP transcription coactivator, and therefore resulting in anoikis. We propose that the detachment-induced Hippo activation and YAP/TAZ inhibition are key events necessary for anoikis induction. Thus, our findings open a new avenue toward anoikis through the Hippo pathway-mediated YAP inhibition evoked by cytoskeleton rearrangements. Such a mechanism suggests a role of the Hippo pathway in clearance of displaced cells to maintain tissue identity during development and in suppression of tumor metastasis. In support of this, reduced Lats $1 / 2$ expression in prostate cancer is found to correlate specifically with metastatic samples, suggesting a clinical relevance of Hippo pathway deregulation in cancer metastasis.

\section{Materials and methods}

\section{Antibodies, plasmids, and other materials}

Anti-CTGF and anti-YAP antibodies were obtained from Santa Cruz Biotechnology. Anti-phospho-YAP (S127), anti-Lats1, antiphospho-Lats1/2(S909/872), anti-phospho-Lats1/2(T1079/999), anti-Mst1, anti-Mst2, anti-PARP, and anti-GST were obtained from Cell Signaling. Anti-TAZ and anti-FAK antibodies were from BD Biosciences. Anti- $\alpha$-Tubulin, anti-Flag, and anti-vinculin antibodies were obtained from Sigma. Anti-HA and anti-Myc antibodies were obtained from Covance. Alexa Fluor 488- or Alexa Fluor 594-conjugated secondary antibodies were obtained from Invitrogen. Horseradish peroxidase-conjugated secondary antibodies were obtained from GE Healthcare.

The pCMV-Flag-YAP wild-type; 5SA, pQCXIH-Myc-YAP wildtype; 5SA, pGEX-KG-YAP, pcDNA3-HA-Lats2; and Flag-Mst2 constructs were described before (Zhao et al. 2007). Phos-tagconjugated acrylamide was purchased from Wako Chemicals. PP2, Y27632, and H1152 were purchased from Calbiochem. PF271 was a gift from Dr. David Schlaepfer. All other chemicals were from Sigma.

\section{Cell culture, transfection, and retroviral infection}

HeLa cells were cultured in DMEM (Invitrogen) containing 10\% FBS (Invitrogen) and $50 \mu \mathrm{g} / \mathrm{mL}$ penicillin/streptomycin (P/S). MCF10A cells were cultured in DMEM/F12 (Invitrogen) supplemented with $5 \%$ horse serum (Invitrogen), $20 \mathrm{ng} / \mathrm{mL}$ EGF, 0.5 $\mu \mathrm{g} / \mathrm{mL}$ hydrocortisone, $10 \mu \mathrm{g} / \mathrm{mL}$ insulin, $100 \mathrm{ng} / \mathrm{mL}$ cholera toxin, and $50 \mu \mathrm{g} / \mathrm{mL} \mathrm{P} / \mathrm{S}$. Immortalized human breast epithelial cells (HMLE) were cultured in MEGM medium (Lonza). Transfection with Lipofectamine was performed according to the manufacturer's instructions.

To generate wild-type or mutant YAP-expressing stable cells, retrovirus infection was performed by transfecting 293 Phoenix retroviral packaging cells with empty vector or pQCXIH-YAP constructs. Forty-eight hours after transfection, retroviral supernatant was supplemented with $5 \mu \mathrm{g} / \mathrm{mL}$ polybrene, filtered through a $0.45-\mu \mathrm{m}$ filter, and used to infect MCF10A or HMLE cells. Thirty-six hours after infection, cells were selected with $200 \mu \mathrm{g} / \mathrm{mL}$ hygromycin (Roche) in culture medium.

\section{Immunofluorescence staining}

Cells were cultured on fibronectin or polylysine-coated coverslips to the appropriate density and treated as indicated in experiments. Cells were fixed with $4 \%$ paraformaldehyde for 15 min and then permeabilized with $0.1 \%$ Triton X-100. After blocking in 3\% BSA for 30 min, slides were incubated with first antibodies diluted in $1 \%$ BSA for $1.5 \mathrm{~h}$ at room temperature or overnight at $4^{\circ} \mathrm{C}$. After washing with PBS, slides were incubated with Alexa Fluor 488- or Alexa Fluor 594-conjugated secondary antibodies (1:1000 dilution) for $1.5 \mathrm{~h}$. The slides were then washed and mounted. For staining of the microtubule, cells were fixed in 
PHEMO fixative $(3.7 \%$ PFA, $0.05 \%$ glutaraldehyde, $0.5 \%$ Triton $\mathrm{X}-100$ in $68 \mathrm{mM}$ PIPES, $25 \mathrm{mM}$ HEPES, $15 \mathrm{mM}$ EGTA, $3 \mathrm{mM}$ $\mathrm{MgCl}_{2}, 10 \%$ dimethylsulfoxide at $\mathrm{pH} 6.8$ ) for $10 \mathrm{~min}$ at room temperature. For staining of F-actin, cells were incubated with $6.6 \mathrm{nM}$ Alexa Fluor 488-phalloidin for $1.5 \mathrm{~h}$ after blocking.

\section{Immunoprecipitation and kinase assay}

For Lats1/2 kinase assays, cells were lysed with lysis buffer (50 mM HEPES at $\mathrm{pH} 7.5,150 \mathrm{mM} \mathrm{NaCl}, 1 \mathrm{mM}$ EDTA, $1 \%$ NP-40, $10 \mathrm{mM}$ pyrophosphate, $10 \mathrm{mM}$ glycerophosphate, $50 \mathrm{mM}$ $\mathrm{NaF}, 1.5 \mathrm{mM}$ Na3VO4, protease inhibitor cocktail [Roche], $1 \mathrm{mM}$ DTT, $1 \mathrm{mM}$ PMSF). Endogenous Lats1 or ectopically expressed Lats2 were immunoprecipitated with anti-Lats1 or anti-HA antibodies, respectively. The immunoprecipitates were washed three times with lysis buffer, and then once with wash buffer $(40 \mathrm{mM}$ HEPES, $200 \mathrm{mM} \mathrm{NaCl}$ ) and once with kinase assay buffer $(30 \mathrm{mM}$ HEPES, $50 \mathrm{mM}$ potassium acetate, $5 \mathrm{mM} \mathrm{MgCl}_{2}$ ). The immunoprecipitates were then subjected to a kinase assay in the presence of $500 \mu \mathrm{M}$ cold ATP, $10 \mu \mathrm{Ci}\left(\gamma_{-}{ }^{32} \mathrm{P}\right)$ ATP, and $1 \mu \mathrm{g}$ GST-YAP expressed and purified from Escherichia coli as substrate. The reaction mixtures were incubated for $30 \mathrm{~min}$ at $30^{\circ} \mathrm{C}$, terminated with SDS sample buffer, and subjected to SDS-PAGE. YAP phosphorylation was detected by a phospho-specific antibody. For detection of YAP upstream kinase activity, $1 \mu \mathrm{g}$ of GST-YAP immobilized on glutathione resin was first incubated with cell lysates for $2 \mathrm{~h}$. The washed resins were then subjected to kinase assay similar to that described above except that GSTYAP was not further supplemented.

\section{Soft agar colony formation assay}

Cells $(5 \times 103)$ were added to $1.5 \mathrm{~mL}$ of growth medium with $0.4 \%$ agarose and layered onto $2 \mathrm{~mL}$ of $0.5 \%$ agarose beds in sixwell plates. Cells were fed with $2 \mathrm{~mL}$ of growth medium every week for $3 \mathrm{wk}$, after which colonies were pictured and counted.

\section{Annexin $V$ staining and flow cytometry}

For anoikis analysis, cells were cultured in attachment or suspension. Cells were then trypsinized and stained with PE Annexin $\mathrm{V}$ and analyzed by flow cytometry using the PE Annexin V Apoptosis Detection kit (BD Biosciences) following the manufacturer's instructions. Data were collected and analyzed on a BD FACSCanto using FACSDiva software.

\section{RNAi}

SMARTpool siRNA oligonucleotides toward human Lats1, Lats2, Mst1, Mst2, and control siRNA toward firefly luciferase were purchased from Dharmacon. siRNAs were transfected into HeLa cells twice with a 24 -h interval. Cells were lysed 48 h post-transfection.

\section{RNA isolation and real-time PCR}

Total RNA was isolated from cultured cells using Trizol reagent (Invitrogen). cDNA was synthesized by reverse transcription using random hexamers and was subjected to real-time PCR with genespecific primers in the presence of SYBR Green (Applied Biosystems). Relative abundance of mRNA was calculated by normalization to hypoxanthine phosphoribosyltransferase 1 (HPRT) mRNA.

\section{Cancer microarray data analysis}

Microarray data profiling mRNA expression of the prostate cancer cohort was downloaded from the Gene Expression Omnibus
(GEO) database (GSE21034) (Taylor et al. 2010). Expression values were log-transformed and normalized to the average of the benign samples. The box plot in Figure $6 \mathrm{C}$ was generated using $\mathrm{R}$.

\section{Acknowledgments}

We thank Drs. Shu Chien and Julie Yi-Shuan Li for suggestions on experiment design, Dr. Ming Yan for assistance on FACS analysis, Dr. Yingzi Yang for Mst1/2 MEF cells, and Dr. Jenna L. Jewell for critical reading of the manuscript. NIH Grant P30 CA23100 covered part of the cost for confocal imaging. This work was supported by grants from the NIH (K.L.G., C.Y.W., and J.D.Y.), CIRM (K.L.G.), and DOD (J.D.Y., PC080665).

\section{References}

Chan EH, Nousiainen M, Chalamalasetty RB, Schafer A, Nigg EA, Sillje HH. 2005. The Ste20-like kinase Mst2 activates the human large tumor suppressor kinase Lats1. Oncogene 24: 2076-2086.

Chan SW, Lim CJ, Chong YF, Venkatesan Pobbati A, Huang C, Hong W. 2011. Hippo pathway-independent restriction of TAZ and YAP by angiomotin. I Biol Chem 286: 7018-7026.

Chiarugi P, Giannoni E. 2008. Anoikis: A necessary death program for anchorage-dependent cells. Biochem Pharmacol 76: $1352-1364$.

Dong J, Feldmann G, Huang J, Wu S, Zhang N, Comerford SA, Gayyed MF, Anders RA, Maitra A, Pan D. 2007. Elucidation of a universal size-control mechanism in Drosophila and mammals. Cell 130: 1120-1133.

Dupont S, Morsut L, Aragona M, Enzo E, Giulitti S, Cordenonsi M, Zanconato F, Le Digabel J, Forcato M, Bicciato S, et al. 2011. Role of YAP/TAZ in mechanotransduction. Nature 474: 179-183.

Fernandez BG, Gaspar P, Bras-Pereira C, Jezowska B, Rebelo SR, Janody F. 2011. Actin-Capping Protein and the Hippo pathway regulate F-actin and tissue growth in Drosophila. Development 138: 2337-2346.

Frantz C, Stewart KM, Weaver VM. 2010. The extracellular matrix at a glance. J Cell Sci 123: 4195-4200.

Grashoff C, Hoffman BD, Brenner MD, Zhou R, Parsons M, Yang MT, McLean MA, Sligar SG, Chen CS, Ha T, et al. 2010. Measuring mechanical tension across vinculin reveals regulation of focal adhesion dynamics. Nature 466: 263-266.

Halder G, Johnson RL. 2011. Hippo signaling: Growth control and beyond. Development 138: 9-22.

Hanahan D, Weinberg RA. 2000. The hallmarks of cancer. Cell 100: $57-70$.

Hao Y, Chun A, Cheung K, Rashidi B, Yang X. 2008. Tumor suppressor LATS1 is a negative regulator of oncogene YAP. J Biol Chem 283: 5496-5509.

Harvey KF, Pfleger CM, Hariharan IK. 2003. The Drosophila Mst ortholog, hippo, restricts growth and cell proliferation and promotes apoptosis. Cell 114: 457-467.

Huang J, Wu S, Barrera J, Matthews K, Pan D. 2005. The Hippo signaling pathway coordinately regulates cell proliferation and apoptosis by inactivating Yorkie, the Drosophila Homo$\log$ of YAP. Cell 122: 421-434.

Jia J, Zhang W, Wang B, Trinko R, Jiang J. 2003. The Drosophila Ste20 family kinase dMST functions as a tumor suppressor by restricting cell proliferation and promoting apoptosis. Genes Dev 17: 2514-2519.

Justice RW, Zilian O, Woods DF, Noll M, Bryant PJ. 1995. The Drosophila tumor suppressor gene warts encodes a homolog of human myotonic dystrophy kinase and is required for the control of cell shape and proliferation. Genes Dev 9: 534-546. 
Kango-Singh M, Nolo R, Tao C, Verstreken P, Hiesinger PR, Bellen HJ, Halder G. 2002. Shar-pei mediates cell proliferation arrest during imaginal disc growth in Drosophila. Development 129: 5719-5730.

Kim NG, Koh E, Chen X, Gumbiner BM. 2011. E-cadherin mediates contact inhibition of proliferation through Hippo signaling-pathway components. Proc Natl Acad Sci 108: 11930-11935.

Lai ZC, Wei X, Shimizu T, Ramos E, Rohrbaugh M, Nikolaidis N, Ho LL, Li Y. 2005. Control of cell proliferation and apoptosis by mob as tumor suppressor, mats. Cell 120: 675-685.

Lai D, Ho KC, Hao Y, Yang X. 2011. Taxol resistance in breast cancer cells is mediated by the hippo pathway component TAZ and its downstream transcriptional targets Cyr61 and CTGF. Cancer Res 71: 2728-2738.

Lee KP, Lee JH, Kim TS, Kim TH, Park HD, Byun JS, Kim MC, Jeong WI, Calvisi DF, Kim JM, et al. 2010. The HippoSalvador pathway restrains hepatic oval cell proliferation, liver size, and liver tumorigenesis. Proc Natl Acad Sci 107: 8248-8253.

Lei QY, Zhang H, Zhao B, Zha ZY, Bai F, Pei XH, Zhao S, Xiong Y, Guan KL. 2008. TAZ promotes cell proliferation and epithelial-mesenchymal transition and is inhibited by the hippo pathway. Mol Cell Biol 28: 2426-2436.

Liu CY, Zha ZY, Zhou X, Zhang H, Huang W, Zhao D, Li T, Chan SW, Lim CJ, Hong W, et al. 2010. The hippo tumor pathway promotes TAZ degradation by phosphorylating a phosphodegron and recruiting the SCF $\beta$-TrCP E3 ligase. I Biol Chem 285: 37159-37169.

Lu L, Li Y, Kim SM, Bossuyt W, Liu P, Qiu Q, Wang Y, Halder G, Finegold MJ, Lee JS, et al. 2010. Hippo signaling is a potent in vivo growth and tumor suppressor pathway in the mammalian liver. Proc Natl Acad Sci 107: 1437-1442.

Mammoto A, Ingber DE. 2009. Cytoskeletal control of growth and cell fate switching. Curr Opin Cell Biol 21: 864-870.

Nishioka N, Inoue K, Adachi K, Kiyonari H, Ota M, Ralston A, Yabuta N, Hirahara S, Stephenson RO, Ogonuki N, et al. 2009. The Hippo signaling pathway components Lats and Yap pattern Tead4 activity to distinguish mouse trophectoderm from inner cell mass. Dev Cell 16: 398-410.

Onder TT, Gupta PB, Mani SA, Yang J, Lander ES, Weinberg RA. 2008. Loss of E-cadherin promotes metastasis via multiple downstream transcriptional pathways. Cancer Res 68: 36453654.

Overholtzer M, Zhang J, Smolen GA, Muir B, Li W, Sgroi DC, Deng CX, Brugge JS, Haber DA. 2006. Transforming properties of YAP, a candidate oncogene on the chromosome 11q22 amplicon. Proc Natl Acad Sci 103: 12405-12410.

Pan D. 2010. The hippo signaling pathway in development and cancer. Dev Cell 19: 491-505.

Pantalacci S, Tapon N, Leopold P. 2003. The Salvador partner Hippo promotes apoptosis and cell-cycle exit in Drosophila. Nat Cell Biol 5: 921-927.

Parsons JT, Horwitz AR, Schwartz MA. 2010. Cell adhesion: Integrating cytoskeletal dynamics and cellular tension. Nat Rev Mol Cell Biol 11: 633-643.

Praskova M, Xia F, Avruch J. 2008. MOBKL1A/MOBKL1B phosphorylation by MST1 and MST2 inhibits cell proliferation. Curr Biol 18: 311-321.

Sansores-Garcia L, Bossuyt W, Wada K, Yonemura S, Tao C, Sasaki H, Halder G. 2011. Modulating F-actin organization induces organ growth by affecting the Hippo pathway. EMBO I 30: 2325-2335.

Schlegelmilch K, Mohseni M, Kirak O, Pruszak J, Rodriguez JR, Zhou D, Kreger BT, Vasioukhin V, Avruch J, Brummelkamp
TR, et al. 2011. Yap1 acts downstream of $\alpha$-catenin to control epidermal proliferation. Cell 144: 782-795.

Silvis MR, Kreger BT, Lien WH, Klezovitch O, Rudakova GM, Camargo FD, Lantz DM, Seykora JT, Vasioukhin V. 2011. $\alpha$-Catenin is a tumor suppressor that controls cell accumulation by regulating the localization and activity of the transcriptional coactivator Yap1. Sci Signal 4: ra33. doi: 10.1126/scisignal.2001823.

Song H, Mak KK, Topol L, Yun K, Hu J, Garrett L, Chen Y, Park O, Chang J, Simpson RM, et al. 2010. Mammalian Mst1 and Mst2 kinases play essential roles in organ size control and tumor suppression. Proc Natl Acad Sci 107: 1431-1436.

Steinhardt AA, Gayyed MF, Klein AP, Dong J, Maitra A, Pan D, Montgomery EA, Anders RA. 2008. Expression of Yes-associated protein in common solid tumors. Hum Pathol 39: $1582-1589$

Tapon N, Harvey KF, Bell DW, Wahrer DC, Schiripo TA, Haber DA, Hariharan IK. 2002. salvador promotes both cell cycle exit and apoptosis in Drosophila and is mutated in human cancer cell lines. Cell 110: 467-478.

Taylor BS, Schultz N, Hieronymus H, Gopalan A, Xiao Y, Carver BS, Arora VK, Kaushik P, Cerami E, Reva B, et al. 2010. Integrative genomic profiling of human prostate cancer. Cancer Cell 18: 11-22.

Udan RS, Kango-Singh M, Nolo R, Tao C, Halder G. 2003. Hippo promotes proliferation arrest and apoptosis in the Salvador/ Warts pathway. Nat Cell Biol 5: 914-920.

Varelas X, Samavarchi-Tehrani P, Narimatsu M, Weiss A, Cockburn K, Larsen BG, Rossant J, Wrana JL. 2010. The crumbs complex couples cell density sensing to Hippodependent control of the TGF- $\beta-S M A D$ pathway. Dev Cell 19: $831-844$.

Wada K, Itoga K, Okano T, Yonemura S, Sasaki H. 2011. Hippo pathway regulation by cell morphology and stress fibers. Development 138: 3907-3914.

Wang W, Huang J, Chen J. 2011. Angiomotin-like proteins associate with and negatively regulate YAP1. I Biol Chem 286: 4364-4370.

Wu S, Huang J, Dong J, Pan D. 2003. hippo encodes a Ste-20 family protein kinase that restricts cell proliferation and promotes apoptosis in conjunction with salvador and warts. Cell 114: 445-456.

Xu T, Wang W, Zhang S, Stewart RA, Yu W. 1995. Identifying tumor suppressors in genetic mosaics: The Drosophila lats gene encodes a putative protein kinase. Development 121: 1053-1063.

Xu MZ, Yao TJ, Lee NP, Ng IO, Chan YT, Zender L, Lowe SW, Poon RT, Luk JM. 2009. Yes-associated protein is an independent prognostic marker in hepatocellular carcinoma. Cancer 115: 4576-4585.

Zender L, Spector MS, Xue W, Flemming P, Cordon-Cardo C, Silke J, Fan ST, Luk JM, Wigler M, Hannon GJ, et al. 2006. Identification and validation of oncogenes in liver cancer using an integrative oncogenomic approach. Cell 125: 12531267.

Zhang J, Ji JY, Yu M, Overholtzer M, Smolen GA, Wang R, Brugge JS, Dyson NJ, Haber DA. 2009. YAP-dependent induction of amphiregulin identifies a non-cell-autonomous component of the Hippo pathway. Nat Cell Biol 11: 14441450 .

Zhang H, Pasolli HA, Fuchs E. 2011. Yes-associated protein (YAP) transcriptional coactivator functions in balancing growth and differentiation in skin. Proc Natl Acad Sci 108: 2270-2275

Zhao B, Wei X, Li W, Udan RS, Yang Q, Kim J, Xie J, Ikenoue T, $\mathrm{Yu}$, Li L, et al. 2007. Inactivation of YAP oncoprotein by the 
Zhao et al.

Hippo pathway is involved in cell contact inhibition and tissue growth control. Genes Dev 21: 2747-2761.

Zhao B, Ye X, Yu J, Li L, Li W, Li S, Lin JD, Wang CY, Chinnaiyan AM, Lai ZC, et al. 2008. TEAD mediates YAPdependent gene induction and growth control. Genes Dev 22: 1962-1971.

Zhao B, Li L, Tumaneng K, Wang CY, Guan KL. 2010. A coordinated phosphorylation by Lats and CK1 regulates YAP stability through SCF( $\beta$-TRCP). Genes Dev 24: 72-85.

Zhao B, Li L, Lu Q, Wang LH, Liu CY, Lei Q, Guan KL. 2011. Angiomotin is a novel Hippo pathway component that inhibits YAP oncoprotein. Genes Dev 25: 51-63.

Zhou D, Conrad C, Xia F, Park JS, Payer B, Yin Y, Lauwers GY, Thasler W, Lee JT, Avruch J, et al. 2009. Mst1 and Mst2 maintain hepatocyte quiescence and suppress hepatocellular carcinoma development through inactivation of the Yap1 oncogene. Cancer Cell 16: 425-438. 


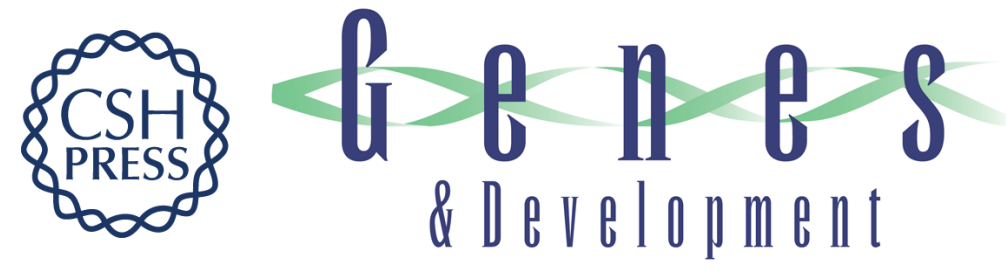

\section{Cell detachment activates the Hippo pathway via cytoskeleton reorganization to induce anoikis}

Bin Zhao, Li Li, Lloyd Wang, et al.

Genes Dev. 2012, 26:

Access the most recent version at doi:10.1101/gad.173435.111

Supplemental
Material http://genesdev.cshlp.org/content/suppl/2012/01/03/26.1.54.DC1

References This article cites 53 articles, 25 of which can be accessed free at:

http://genesdev.cshlp.org/content/26/1/54.full.html\#ref-list-1

License

Email Alerting Receive free email alerts when new articles cite this article - sign up in the box at the top

Service right corner of the article or click here.

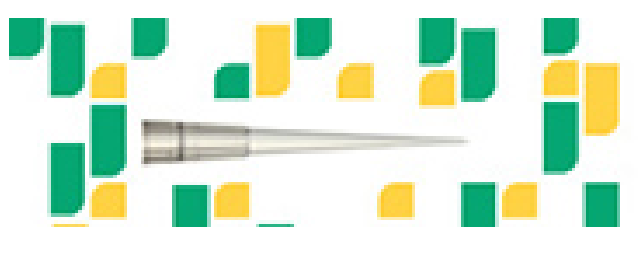

Focused on your science. 